\title{
Modeling the Critical and Phase Equilibrium Properties of Pure Fluids and Mixtures with the Crossover Cubic-Plus-Association Equation of State
}

\author{
Vinhal, Andre P. C. M.; Yan, Wei; Kontogeorgis, Georgios M.
}

Published in:

Journal of Chemical and Engineering Data

Link to article, DOI:

10.1021/acs.jced.9b00492

Publication date:

2020

Document Version

Peer reviewed version

Link back to DTU Orbit

Citation (APA):

Vinhal, A. P. C. M., Yan, W., \& Kontogeorgis, G. M. (2020). Modeling the Critical and Phase Equilibrium

Properties of Pure Fluids and Mixtures with the Crossover Cubic-Plus-Association Equation of State. Journal of Chemical and Engineering Data, 65(3), 1095-1107. https://doi.org/10.1021/acs.jced.9b00492

\section{General rights}

Copyright and moral rights for the publications made accessible in the public portal are retained by the authors and/or other copyright owners and it is a condition of accessing publications that users recognise and abide by the legal requirements associated with these rights.

- Users may download and print one copy of any publication from the public portal for the purpose of private study or research.

- You may not further distribute the material or use it for any profit-making activity or commercial gain

- You may freely distribute the URL identifying the publication in the public portal 


\title{
Modelling the Critical and Phase Equilibrium Properties of Pure Fluids and Mixtures with the Crossover Cubic-Plus-Association Equation of State
}

\author{
Andre P. C. M. Vinhal ${ }^{1}$, Wei Yan $^{2}$, Georgios M. Kontogeorgis ${ }^{1, *}$ \\ ${ }^{1}$ Center for Energy Resources Engineering (CERE), Department of Chemical and Biochemical Engineering, Technical \\ University of Denmark, DK-2800 Kgs. Lyngby, Denmark \\ ${ }^{2}$ Center for Energy Resources Engineering (CERE), Department of Chemistry, Technical University of Denmark, DK-2800 \\ Kgs. Lyngby, Denmark
}

*Corresponding author. E-mail: gk@kt.dtu.dk

\begin{abstract}
The Cubic-Plus-Association $(C P A)$ Equation of State $(E O S)$ is unable to correctly describe the vapor-liquid critical behavior of pure fluids and mixtures due to its classical behavior. In fact, the traditional parametrization procedure, i.e. to match the saturated pressure and liquid density curves far from the critical point, causes an over-prediction of the critical pressures and temperatures of pure components. Besides, the deviations with respect to experimental data are even larger for systems containing hydrogen-bonding species, in comparison to systems composed of non-associating molecules. In order to improve the representation of the thermodynamic properties of fluids in near-critical regions with $C P A$, we have applied White's recursive procedure to introduce density fluctuations in the classical model, allowing the correct description of the non-analytical behavior of real fluids close to the critical point. The resulting model, i.e., the Crossover CPA (CCPA) EoS, is capable of accurately representing the phase behavior of fluids, specifically normal alkanes and alcohols, carbon dioxide, water, as well as some of their binary mixtures, far away from and close to the critical region. This is shown by the comparison of the results obtained from $C C P A$ and classical $E o S$ with the experimental data.
\end{abstract}

\section{Introduction}

Industrial processes in the field of chemical and petroleum engineering usually require detailed knowledge of the phase behavior and thermophysical properties of the fluids in order to achieve an efficient design of equipment and operation control. ${ }^{1}$ Because of the difficulties involving the acquirement of experimental data for different operational conditions, the simulation of industrial processes usually relies on the accuracy of a thermodynamic model to estimate the properties of fluids. As an example, compositional reservoir simulators use cubic Equations of State $(E o S)$, due to their simplicity and capacity to correctly describe the phase behavior of pure fluids and mixtures containing hydrocarbons. ${ }^{2}$ Nevertheless, challenging situations are observed when theoretical equations of state are applied to represent systems containing highly non-ideal species, e.g. associating molecules, ${ }^{3}$ or conditions close to the critical point, due to the non-analytical and asymptotic behavior of real fluids. ${ }^{4,5}$

Regarding the first issue, it is known in the literature that the traditional cubic EoS are often incapable of satisfactorily represent the phase behavior of complex systems containing hydrogen bonding species. ${ }^{6,7}$ In order to improve the representation of such systems, the Cubic-
Plus-Association (CPA) $\mathrm{EoS}^{8}$ has been developed and this model combines the Soave-Redlich-Kwong (SRK) ${ }^{9}$ equation with Wertheim's association term, ${ }^{10}$ thus resulting in a model that is based on a simple equation, which is widely used in the petroleum industry, but also describes the hydrogen-bonding between molecules of a system using the perturbation theory. ${ }^{3}$ Nevertheless, due to its classical behavior and the traditional parametrization procedure, i.e. to match saturated pressure and liquid density curves far from the critical point, ${ }^{11}$ this approach is particularly successful for representing the behavior of associating systems far from the critical point, where the fluctuations of the order parameter are negligible. ${ }^{12}$

In the vicinity of the critical point, the thermodynamic properties of fluids cannot be accurately described by classical equations of state $(E o S) .{ }^{13}$ The classical formulations neglect the fluctuations in the order parameter associated with the critical phase transition, hence they predict an analytical behavior of the systems close to the critical point, leading to incorrect estimation of the critical exponents and other properties. ${ }^{14}$ On the other hand, far away from the critical point, where the inhomogeneities are negligible, ${ }^{12}$ classical EoS yield a quantitative representation of the phase behavior of fluids and represent an important tool 
for engineering calculations. Therefore, a model that is capable of describing the global phase behavior of thermodynamic systems, i.e. far away and close to the critical point, is of great scientific and technical relevance. ${ }^{15}$

Several efforts have been made over the last three decades towards the development of a global EoS with a crossover treatment that corrects the classical behavior in the critical region. This correction is normally done by taking into account the fluctuations of the order parameter, which for the vapor-liquid transition is density, using methods based on the renormalization group $(R G)$ theory. ${ }^{16-19}$ The purpose of a $R G$ transformation is to reduce the degrees of freedom of a system by renormalizing its Hamiltonian, that is, to integrate out the microscopic degrees of freedom. ${ }^{20}$ One of the main crossover approaches stems from the LandauGinzburg-Wilson theory of the critical phenomena. ${ }^{12,13,21-}$ ${ }^{26}$ In this case, the nonlinear RG equations are solved by mapping the solution of the renormalized to the classical equation at a cutoff point. The application of this procedure is done by decomposing the system's free energy into an analytical and a non-analytical part, in which the asymptotic critical scaling laws are explicitly incorporated using the crossover transformations. The second main approach was developed by White and coworkers, ${ }^{27-30}$ and has been applied in many different works in the literature. ${ }^{31-38}$ It is an expansion of Wilson's phase-space cell approximation. ${ }^{39}$ The idea behind this method is to reduce the degrees of freedom of the system using a series of renormalized Hamiltonians, in which the fluctuations of the order parameter are averaged. The recursive expressions allow the calculation of explicit values for the critical exponents.

In this work, we use White's procedure to introduce density fluctuations into the classical CPA EoS. The resulting thermodynamic model takes into account hydrogen bonding between molecules and, due to the recursive method to correct the free energy density of the system, it is also capable of representing the behavior of real fluids close to and far away from the critical point. The developed is referred to as the crossover $C P A$ ( $C C P A) E o S$. Although the CCPA EoS has been used in the representation of pure species and binary alkanol/alkane mixtures, ${ }^{35,36}$ it has not been applied in the evaluation of both subcritical calculations, including vapor-liquid (VLE) and liquid-liquid ( $L L E)$, and critical conditions. Besides, this work also deals with the representation of the critical locus of cross-associating and solvating binary mixtures.

The extension of the model for representing the phase behavior of mixtures is done using the isomorphism assumption, which means that the thermodynamic potential of a mixture is considered to have the same universal form as a one-component fluid. Additionally, the mole fractions are used as independent variables instead of the chemical potential. ${ }^{23}$

In the next section, we present the equations concerning the thermodynamic models utilized in this work. The following sections contain the procedure for obtaining the parameters for pure components and binary mixtures as well as the results, and the last section summarizes of the findings of this work.

\section{The CCPA EoS}

As mentioned in the previous section, the CPA EOS is a combination of the $S R K$ model and the association term from Wertheim's theory. The pressure explicit expression of this equation is given by the expression:

$P=\frac{R T}{v-b}-\frac{a}{v(v+b)}-\frac{1}{2} \frac{R T}{v}\left(1+\rho \frac{\partial \ln g}{\partial \rho}\right) \sum_{i} x_{i} \sum_{A_{i}}\left(1-X_{A_{i}}\right)$

where $P$ is pressure, $R$ is the universal gas constant; $v$ molar volume, $\rho$ is the molar density, $T$ is temperature and the term $X_{A_{i}}$ represents the molar fraction of sites $A$ of the molecule $i$ that does not bond to other active sites. 40 The term $X_{A_{i}}$ is related to the association strength, $\Delta^{A_{i} B_{j}}$, between two sites belonging to two different molecules and is calculated by:

$X_{A_{i}}=\left(1+\rho \sum_{i} x_{i} \sum_{B_{j}} X_{B_{j}} \Delta^{A_{i} B_{j}}\right)^{-1}$

The association strength is given by:

$\Delta^{A_{i} B_{j}}=g(\rho)\left[\exp \left(\frac{\varepsilon^{A_{i} B_{j}}}{R T}-1\right)\right] b_{i j} \beta^{A_{i} B_{j}}$

In this work we used the simplified radial distribution function, ${ }^{41,42} g(\rho)$, which is evaluated at contact distance and is determined by the expression:

$g(\rho)=\left[1-1.9\left(\frac{b \rho}{4}\right)\right]^{-1}$

The constant $a$ from the attraction term of the $S R K$ equation is dependent on temperature in the form:

$a=a_{0}\left\{1+c_{1}\left[1-\left(\frac{T}{T_{c}}\right)^{0.5}\right]\right\}^{2}$

The application of the CPA EOS in the calculation of the thermodynamic properties requires the estimation of the pure component parameters which are $a_{0}, b, c_{1}, \varepsilon^{A_{i} B_{j}}$ and $\beta^{A_{i} B_{j}}$ for associating compounds, but reduce to the first three in the case of inert species. Additionally, the model is dependent on the association scheme. In this 
work, we have used the 2B scheme for alcohols and 4C for water, following Huang and Radosz notation. ${ }^{40}$

In the case of mixtures, we used the classical van der Waals mixing rules for the parameters in the attractive and repulsive terms, as described by the following expressions:

$a=\sum_{i=1}^{n} \sum_{j=1}^{n} x_{i} x_{j} a_{i j}$

$b=\sum_{i=1}^{n} x_{i} b_{i}$

where $\mathrm{x}_{\mathrm{i}}$ is the fraction of component $\mathrm{i}$ in a particular phase.

A binary interaction parameter in the cross-energy term was also used for some of the mixtures studied in this work:

$a_{i j}=\left(a_{i i} a_{j j}\right)^{\frac{1}{2}}\left(1-k_{i j}\right)$

where $k_{i j}$ is the binary interaction coefficient. For the cross-association between two associating components, the following combining rules were used:

$\varepsilon^{A_{i} B_{j}}=\frac{\varepsilon^{A_{i} B_{i}}+\varepsilon^{A_{j} B_{j}}}{2}$

$\beta^{A_{i} B_{j}}=\sqrt{\beta^{A_{i} B_{i}} \beta^{A_{j} B_{j}}}$

Finally, another possibility of cross-association occurs between carbon dioxide and a hydrogen-bonding molecule, due to the induced association between the inert and the associating species. These systems are called solvating mixtures, and a modified $C R I$ rule can be used to improve the representation of the thermodynamic properties. ${ }^{3}$ In the modified $C R 1$ rule the cross-association energy parameter is given by eq 9 , while the cross-association volume is obtained from fitting experimental data.

The CPA EoS is a model that successfully represents systems containing hydrogen bonding species. ${ }^{3}$ Nonetheless, like any other classical model, it fails to describe the near-critical behavior of real fluids, since it does not account for the long-range fluctuations that are significant in the vicinities of the critical point. ${ }^{12}$ Therefore, we applied White's recursive procedure in which the long $\left(\Omega_{n}^{l}\right)$ and short $\left(\Omega_{n}^{s}\right)$ range interactions are added to the free energy density $(f)$ via the recursive procedure described by the following expressions:

$f=f_{0}^{C P A}+\sum_{n} \delta f_{n}$

$\delta f_{n}=-K_{n} \ln \left(\Omega_{n}^{s} / \Omega_{n}^{l}\right)$

$$
\begin{aligned}
& K_{n}=k_{b} T / 2^{3 n} L^{3} \\
& \Omega_{n}^{\lambda}=\int_{0}^{\min \left(\rho, \rho^{\max }-\rho\right)} d y \exp \left(-G_{n}^{\lambda} / K_{n}\right) \\
& G_{n}^{\lambda}(\rho, y)=0.5\left[\bar{f}_{n}^{\lambda}(\rho+y)+\bar{f}_{n}^{\lambda}(\rho-y)-2 \bar{f}_{n}^{\lambda}(\rho)\right] \\
& \bar{f}_{n}^{l}(\rho)=f_{n-1}(\rho)+\alpha \rho^{2} \\
& \bar{f}_{n}^{s}(\rho)=f_{n-1}(\rho)+2^{-2 n} \phi \alpha \rho^{2}
\end{aligned}
$$

where $k_{b}$ is the Boltzmann's constant, the superscript $\lambda$ refers to both the long-range $(l)$ and short-range $(s)$ correlations, while $\phi$ and $L$ are two additional parameters. The first is the inverse of the initial shortest wavelength and the second is the cutoff length. ${ }^{27}$

The calculation of the free energy corrections due to the fluctuations in the order parameter requires the use of an initial free energy expression that accounts for the short-range interactions only. ${ }^{27}$ The zero-order solution of the Helmholtz energy density for $C P A$, i.e. $f_{0}^{C P A}$, is given by the expression:

$$
\begin{aligned}
& f_{0}^{C P A}=\frac{-R T \ln (v-b)}{v}-\frac{a}{b v} \ln \left(\frac{v-b}{v}\right)+\frac{R T}{v} \sum_{i} x_{i}\left[\sum _ { A _ { i } } \left(\ln X_{A_{i}}-\right.\right. \\
& \left.\left.\frac{X_{A_{i}}}{2}+\frac{1}{2}\right)\right]+\alpha \rho^{2}
\end{aligned}
$$

where $\alpha \rho^{2}$ is the correction of the attractive part calculated by the expression:

$-\alpha \rho^{2}=-0.5 a \rho^{2}$

The constant $a$ in the previous expression is calculated by eq 5 . This is the same expression used in the crossover approach for the $S R K$ model and it is explained in detail in our recent work. ${ }^{31}$ It is important to note that, for systems composed entirely of nonassociating species, the association term from Wertheim's theory is discarded and the CPA EOS reduces to $S R K$. $^{3}$ Similarly, eq 18 of the CCPA model becomes equal to the expression for the crossover SRK EoS $(C S R K),{ }^{31}$ i.e. $C C P A$ reduces to $C S R K$.

In addition to the eq 11 to eq 19 , the application of the method requires the determination of the number of iterations in the mathematical procedure $(n)$, which were chosen to be 5 , as well as the number of density steps $(\Delta \rho)$ used in the numerical integration of eq 14 , which were chosen to be 500. In both cases, the choice for the number of iteration and steps were based on other studies. ${ }^{31-38}$ Finally, $f(\rho)$ is obtained with a spline function.

The recursive procedure also requires mixing rules for the additional parameters. Following the isomorphism assumption, the expressions are given by: 
$L^{3}=\sum x_{i} L_{i}^{3}$

$\phi=x_{i} \phi_{i}$

The model summarized by the equations presented in this section was applied in the calculation of the critical and phase equilibrium and critical properties of pure fluids and binary mixtures. The estimated pure component parameters and the comparison with experimental data, as well as the underlying classical model, are shown in the following section.

\section{Application of $C C P A$ to pure fluids}

The application of the $C C P A$ in the representation of the thermodynamic properties of pure fluids and mixtures require the estimation of the pure component parameters, i.e. $a_{0}, b, c_{1}, \varepsilon^{A_{i} B_{j}}$ and $\beta^{A_{i} B_{j}}$ for the classical $E o S$ and $L$, and $\phi$ for the recursive procedure.

The parameters were estimated by fitting the experimental saturated data: vapor pressure and saturated liquid and vapor densities; as well as the critical temperature, pressure and volume of the compounds. The following equation shows the objective function used in the optimization procedure:
O.F. $=\sum_{i=1}^{N}\left|\frac{P_{\text {sat }}^{\text {exp }}-P_{\text {sat }}^{\text {calc }}}{P_{\text {sat }}^{\text {exp }}}\right|+\sum_{i=1}^{N}\left|\frac{v_{\text {liq }}^{\text {exp }}-v_{\text {liq }}^{\text {calc }}}{v_{\text {liq }}^{\text {liq }}}\right|+$
$\sum_{i=1}^{N}\left|\frac{v_{\text {vap }}^{\text {exp }}-v_{\text {vap }}^{\text {calc }}}{v_{\text {vap }}^{\text {exp }}}\right|+\left|\frac{P_{c}^{\text {exp }}-P_{c}^{\text {calc }}}{P_{c}^{\text {exp }}}\right|+\left|\frac{T_{c}^{\text {exp }}-T_{c}^{\text {calc }}}{T_{c}^{\text {exp }}}\right|+\left|\frac{v_{c}^{\text {exp }}-v_{c}^{\text {calc }}}{v_{c}^{\text {exp }}}\right|$ (22)

where the superscripts exp and calc represent the experimental and calculated properties, respectively; while the subscripts $P_{\text {sat }}$ is the saturated pressure, $v_{\text {liq }}$ and $v_{\text {vap }}$ is the saturated liquid and vapor densities, and the subscript $c$ stands for the critical properties. The range of reduced temperatures used in the calculations of the saturated properties was from 0.5 to 0.99 .

Table 1 shows the optimum parameters obtained for the normal alkanes studied in this work, while Table 2 shows the parameters for the normal alcohols. Table 3 contains the values of the optimum parameters for carbon dioxide and water. The three tables also exhibit the deviations from experimental saturated and critical properties, where it is possible to see that all the properties are precisely described by the model. It should be noted that the parameter $\phi$ for the n-alkanes, carbon dioxide and water was kept constant and equal to 2 , while for the alcohols and water it was used as an adjustable parameter.

Table 1: Parameters of $C C P A$ for methane to n-decane and the deviations from experimental saturated and critical data.

\begin{tabular}{|c|c|c|c|c|c|c|c|c|c|c|}
\hline \multirow{3}{*}{ Substance } & \multicolumn{4}{|c|}{ Parameters } & \multicolumn{6}{|c|}{ Deviations } \\
\hline & $a_{0}$ & $b$ & $c$ & $L$ & $\Delta P_{\text {sat }} *$ & $\Delta v_{l i q} *$ & $\Delta v_{v a p} *$ & $\Delta P_{c}{ }^{*}$ & $\Delta T_{c}{ }^{*}$ & $\Delta v_{c}{ }^{*}$ \\
\hline & $\left(\frac{\text { bar.cm }}{m o l}\right)$ & $\left(\frac{\mathrm{cm}^{3}}{\mathrm{~mol}}\right)$ & $(-)$ & $(\AA)$ & (\%) & (\%) & (\%) & $(\%)$ & $(\%)$ & $(\%)$ \\
\hline $\mathrm{C} 1$ & 2.317 & 28.20 & 0.3913 & 4.345 & 2.5 & 1.2 & 3.4 & 0.01 & 0.06 & 0.05 \\
\hline $\mathrm{C} 2$ & 5.585 & 42.44 & 0.5242 & 4.950 & 2.5 & 1.5 & 2.1 & 0.01 & 0.19 & 2.0 \\
\hline $\mathrm{C} 3$ & 9.214 & 57.76 & 0.6167 & 5.353 & 2.5 & 1.5 & 2.3 & 0.03 & 0.48 & 0.77 \\
\hline $\mathrm{C} 4$ & 13.41 & 73.11 & 0.6906 & 5.694 & 2.0 & 1.7 & 2.4 & 0.02 & 0.67 & 0.10 \\
\hline $\mathrm{C} 5$ & 18.45 & 90.63 & 0.7501 & 6.073 & 2.3 & 1.5 & 1.6 & 0.01 & 0.49 & 1.2 \\
\hline $\mathrm{C} 6$ & 23.55 & 106.4 & 0.8137 & 6.23 & 2.3 & 1.5 & 1.8 & 0.20 & 0.56 & 1.3 \\
\hline $\mathrm{C} 7$ & 29.34 & 125.0 & 0.8954 & 6.581 & 2.2 & 1.5 & 1.4 & 0.21 & 0.71 & 3.1 \\
\hline $\mathrm{C} 8$ & 35.26 & 141.8 & 0.9612 & 6.731 & 2.2 & 1.7 & 1.2 & 0.06 & 0.73 & 4.5 \\
\hline C9 & 41.45 & 159.7 & 1.025 & 6.941 & 2.2 & 1.7 & 1.2 & 0.14 & 0.75 & 5.0 \\
\hline $\mathrm{C} 10$ & 48.31 & 178.5 & 1.078 & 7.176 & 2.2 & 1.4 & 1.2 & 0.78 & 0.60 & 5.5 \\
\hline Average & - & - & - & - & 2.3 & 1.5 & 1.9 & 0.15 & 0.52 & 2.4 \\
\hline
\end{tabular}

*Average Absolute Deviation $(\mathrm{AAD} \%)=(1 / n)\left(\sum\left|X^{\text {calc }}-X^{\text {exp }}\right| / X^{\text {exp }}\right) * 100$ 
Table 2: Parameters of $C C P A$ for methanol to n-octanol and the deviations from experimental saturated and critical data.

\begin{tabular}{|c|c|c|c|c|c|c|c|c|c|c|c|c|c|}
\hline \multirow{3}{*}{ Substance } & \multicolumn{7}{|c|}{ Parameters } & \multicolumn{6}{|c|}{ Deviations } \\
\hline & & & $c$ & $\frac{\varepsilon}{R}$ & $\beta$ & $L$ & $\phi$ & $\Delta P_{\text {sat }}$ & $\Delta v_{l i q}$ & $\Delta v_{v a p}$ & $\Delta P_{c}$ & $\Delta T_{c}$ & $\Delta v_{c}$ \\
\hline & $\left(\frac{\text { bar.cm }}{\text { mol }}\right)$ & $\left(\frac{\mathrm{cm}^{3}}{\mathrm{~mol}}\right)$ & $(-)$ & $(K)$ & $(-)$ & $(\AA)$ & $(-)$ & (\%) & $(\%)$ & $(\%)$ & $(\%)$ & $(\%)$ & $(\%)$ \\
\hline $\mathrm{C} 1 \mathrm{OH}$ & 4.091 & 30.95 & 0.4430 & 2935 & 0.0166 & 5.6229 & 0.585 & 1.67 & 3.75 & 1.08 & 0.01 & 0.17 & 0.00 \\
\hline $\mathrm{C} 2 \mathrm{OH}$ & 8.037 & 48.04 & 0.6820 & 2739 & 0.0081 & 5.4745 & 0.747 & 0.93 & 1.24 & 1.17 & 0.72 & 0.13 & 0.74 \\
\hline $\mathrm{C} 3 \mathrm{OH}$ & 12.59 & 63.54 & 0.8855 & 2414 & 0.0076 & 5.4617 & 1.202 & 2.00 & 1.43 & 2.15 & 0.00 & 0.70 & 0.00 \\
\hline $\mathrm{C} 4 \mathrm{OH}$ & 17.19 & 78.34 & 0.9716 & 2305 & 0.0063 & 5.6365 & 1.457 & 3.12 & 2.94 & 4.13 & 0.01 & 1.12 & 0.12 \\
\hline $\mathrm{C} 5 \mathrm{OH}$ & 21.33 & 96.92 & 0.9444 & 2552 & 0.0060 & 6.2664 & 1.527 & 3.77 & 3.32 & 2.93 & 0.06 & 1.36 & 0.25 \\
\hline $\mathrm{C} 6 \mathrm{OH}$ & 27.10 & 113.2 & 0.9741 & 2487 & 0.0057 & 6.4820 & 1.576 & 5.61 & 3.81 & 2.65 & 0.00 & 0.97 & 0.00 \\
\hline $\mathrm{C} 7 \mathrm{OH}$ & 31.78 & 128.2 & 0.9494 & 2748 & 0.0040 & 6.6191 & 1.638 & 5.18 & 2.09 & 4.23 & 0.02 & 1.34 & 0.00 \\
\hline $\mathrm{C} 8 \mathrm{OH}$ & 38.70 & 145.0 & 1.0389 & 2574 & 0.0037 & 6.8000 & 1.695 & 5.60 & 4.19 & 2.67 & 0.00 & 1.03 & 0.00 \\
\hline Average & - & - & - & - & - & - & - & 3.5 & 2.8 & 2.6 & 0.10 & 0.85 & 0.1 \\
\hline
\end{tabular}

Table 3: Parameters of $C C P A$ for carbon dioxide and water and the deviations from experimental saturated and critical data.

\begin{tabular}{|c|c|c|c|c|c|c|c|c|c|c|c|c|}
\hline \multirow{3}{*}{ Substance } & \multicolumn{6}{|c|}{ Parameters } & \multicolumn{6}{|c|}{ Deviations } \\
\hline & $a_{0}$ & & $c$ & $\frac{\varepsilon}{R}$ & $\beta$ & $L$ & $\Delta P_{\text {sat }}$ & $\Delta v_{l i q}$ & $\Delta v_{\text {vap }}$ & $\Delta P_{c}$ & $\Delta T_{c}$ & $\Delta v_{c}$ \\
\hline & $\left(\frac{\text { bar.cm }}{\text { mol }}\right)$ & $\left(\frac{\mathrm{cm}^{3}}{\mathrm{~mol}}\right)$ & $(-)$ & $(K)$ & $(-)$ & $(\AA)$ & (\%) & $(\%)$ & $(\%)$ & (\%) & $(\%)$ & (\%) \\
\hline $\mathrm{CO}_{2}$ & 3.580 & 26.91 & 0.6503 & - & - & 4.0528 & 1.71 & 2.41 & 1.77 & 0.01 & 0.15 & 0.20 \\
\hline $\mathrm{H}_{2} \mathrm{O}$ & 1.228 & 14.51 & 0.6736 & 2003 & 0.0692 & 5.7000 & 1.83 & 1.08 & 4.23 & 0.53 & 0.52 & 8.70 \\
\hline Average & - & - & - & - & - & - & 1.8 & 1.7 & 3.0 & 0.27 & 0.33 & 4.4 \\
\hline
\end{tabular}

Table 4 shows the average deviations of the saturated and critical properties calculated with the classical $C P A$ with the parameters taken from the literature. ${ }^{42,43}$ The comparison of Tables 1 to 4 shows that although there is an increase in the deviations related to the saturated properties, only the crossover model is capable of simultaneously representing the critical and phase equilibrium properties.

Table 4: Overall AAD calculated with the CPA EoS for the normal alkanes and alcohols studied in this work.

\begin{tabular}{cccccccc}
\hline & \multicolumn{6}{c}{ Deviations } \\
\cline { 2 - 8 } Substance & $\Delta P_{\text {sat }}$ & $\Delta v_{\text {liq }}$ & $\Delta v_{\text {vap }}$ & $\Delta P_{c}$ & $\Delta T_{c}$ & $\Delta v_{c}$ \\
& $(\%)$ & $(\%)$ & $(\%)$ & $(\%)$ & $(\%)$ & $(\%)$ \\
\cline { 4 - 8 } & & 0.90 & 1.50 & 2.90 & 13.7 & 2.20 & 11.8 \\
$\mathrm{HC}$ & & 1.90 & 1.20 & 4.40 & 25.0 & 3.20 & 8.70 \\
$\mathrm{CO}_{2}$ & & 0.30 & 1.64 & 6.45 & 1.91 & 11.3 & 11.2 \\
$\mathrm{H}_{2} \mathrm{O}$ & & 0.80 & 2.08 & 5.26 & 5.26 & 38.1 & 1.03 \\
\hline
\end{tabular}

In addition to the calculations of the deviations of the critical and saturated properties, the saturated pressure and the coexisting diagrams are shown graphically for the normal alkanes (Figure 1) and alcohols (Figure 2), as well as carbon dioxide and water (Figure 3). The figures also contain the results obtained with the classical $S R K$ and $C P A$. The plots indicate that both SRK and CCPA are capable of describing the critical temperature and pressure of the pure components, while $C P A$ overpredicts it. On the other hand, the classical and crossover $C P A$ $E o S$ superior to the cubic model in the representation of the properties of the components far away from the critical point. The introduction of the fluctuations in the classical CPA EOS corrects the overprediction of the critical point and improves the simulations of coexistence curve in the vicinity of the critical point, as the crossover model yields better representation than the cubic one for the near-critical and critical volume. The comparison of Figure 1 to the other two shows that the application of the recursive procedure is more important for associating components, since the deviations for non-associating species are less pronounced. 


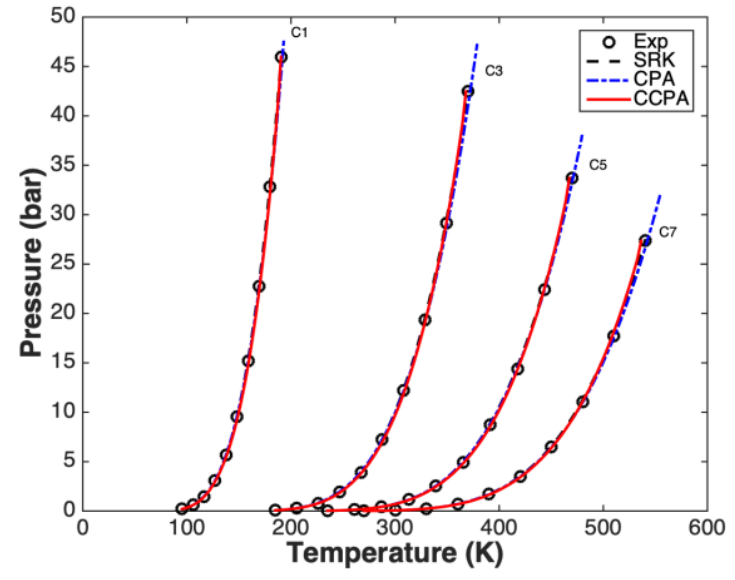

(a)

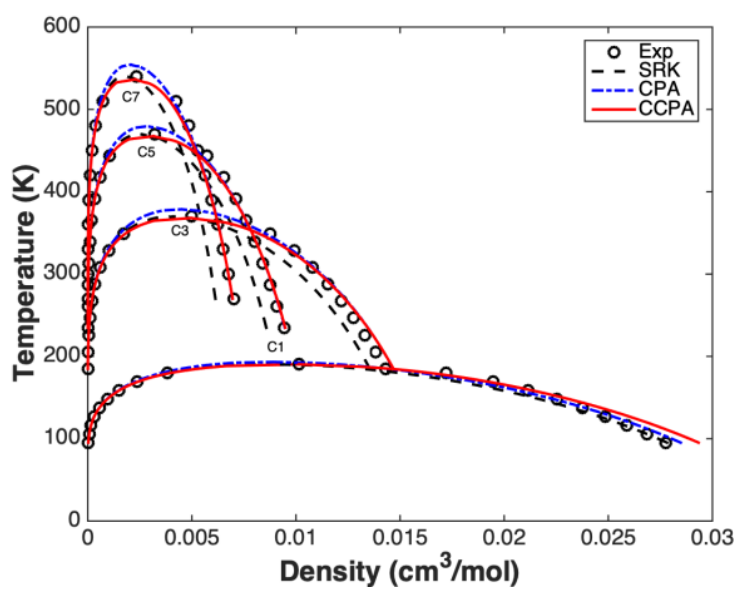

(b)

Figure 1: Saturation pressure as a function of temperature (a) and temperature as a function of the coexisting phases density (b) for selected n-alkanes (from methane to ndecane). Experimental data (open circles) taken from the $D I P P R$ database, ${ }^{55}$ while the dashed black lines, dashdotted blue lines and solid red lines correspond to $S R K$, $C P A$ and $C C P A$, respectively.

The discrepancy in the prediction of the critical point with the classical model is better observed in Figure 4, which shows the critical temperatures, pressures and densities of the substances studied in this work as a function of the molecular weight. The comparison of the predictions obtained from the classical and crossover $C P A E O S$ indicates that the introductions of the fluctuations in the classical $E o S$ are especially useful for correcting the critical pressures and densities, since there is only slight overprediction of the experimental critical temperature data. Additionally, it is possible to observe that the association term deteriorates the description of the critical pressure, since the comparison of the results for alcohols and hydrocarbons with experimental data shows that the hydrogen bonding substances result in a much larger deviation in terms of this property, and this is reduced as the carbon chain length increases, weakening the association between molecules.

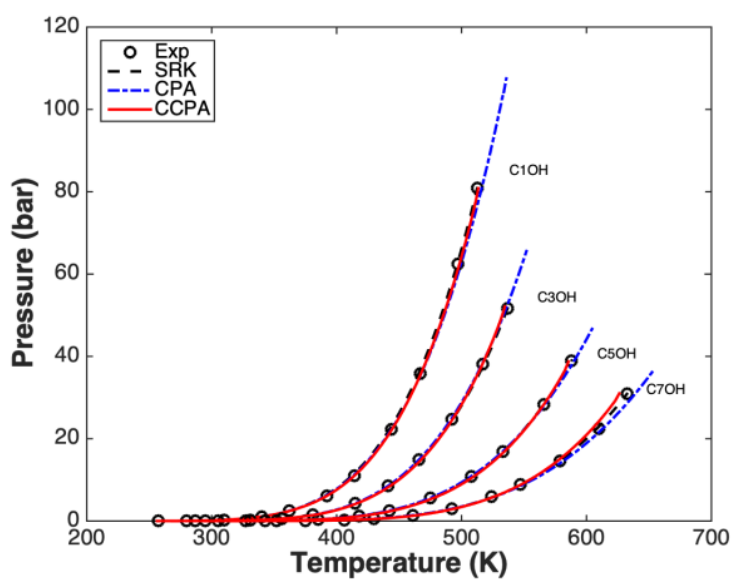

(a)

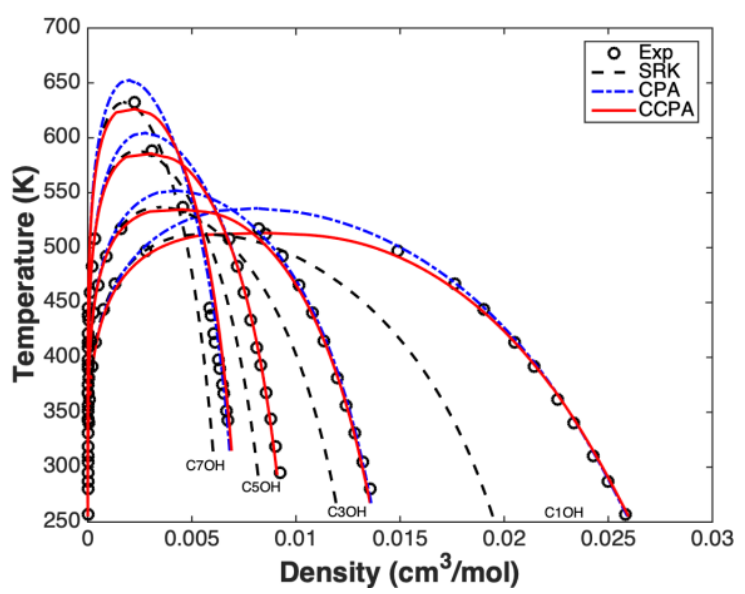

(b)

Figure 2: Saturation pressure as a function of temperature (a) and temperature as a function of the coexisting phases density (b) for selected n-alkanes (from methane to $n$ decane). Experimental data (open circles) taken from the $D I P P R$ database, ${ }^{55}$ while the dashed black lines, dashdotted blue lines and solid red lines correspond to $S R K$, $C P A$ and $C C P A$, respectively.

Another important test, in order to evaluate the capability of the crossover model to describe the nonanalytical nature of the thermodynamic properties in the vicinity of the critical point, is the calculation of the critical exponents. Figure 5 shows the plots of the 
difference between the saturated liquid and vapor densities divided by the critical density as a function of the dimensionless distance to the critical temperature, as well as the dimensionless distance to the critical chemical potential as a function of the dimensionless distance to the critical density. In the first case the slope of the curve represents the critical exponent $\beta$, while in the second case the slope represents the critical exponent $\delta$. The classical values for these two exponents are 0.5 and 3.0, while the ones obtained in our calculations are 0.370 and 4.668 , respectively, which are closer to the universal values 0.326 and $4.8 .{ }^{44}$

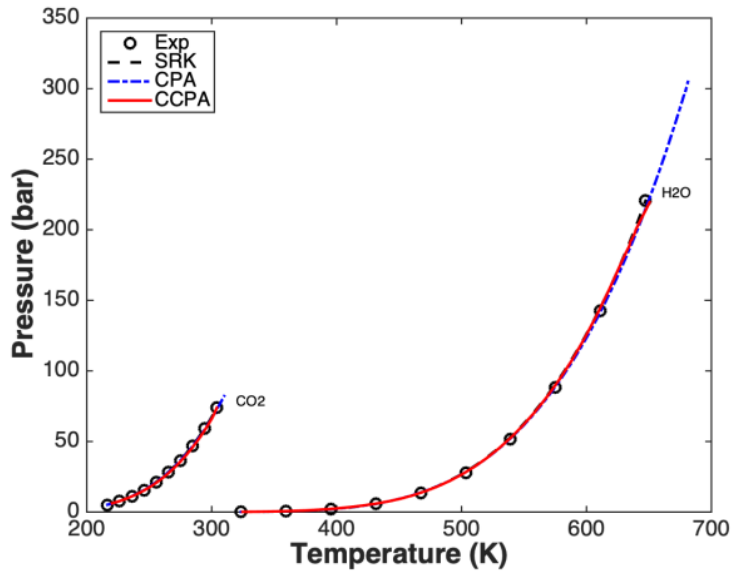

(a)

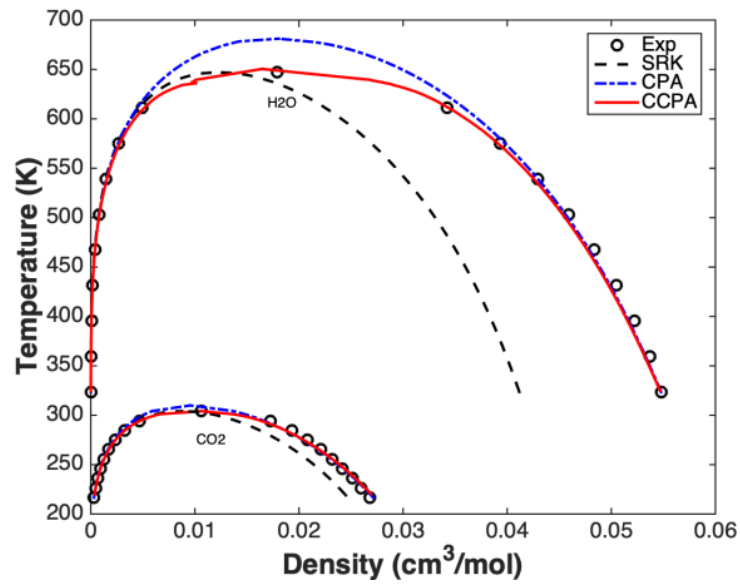

(b)

Figure 3: Saturation pressure as a function of temperature (a) and temperature as a function of the coexisting phases density (b) for carbon dioxide and water. Experimental data (open circles) taken from the DIPPR database, ${ }^{55}$ while the dashed black lines, dash-dotted blue lines and solid red lines correspond to SRK, CPA and CCPA, respectively.
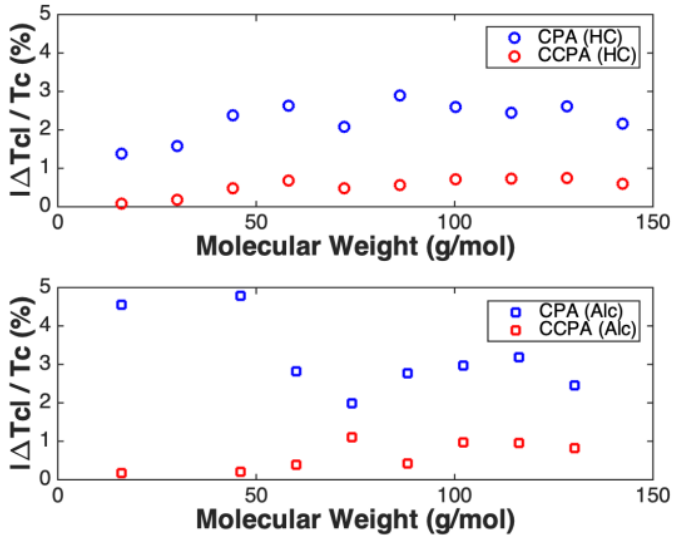

(a)
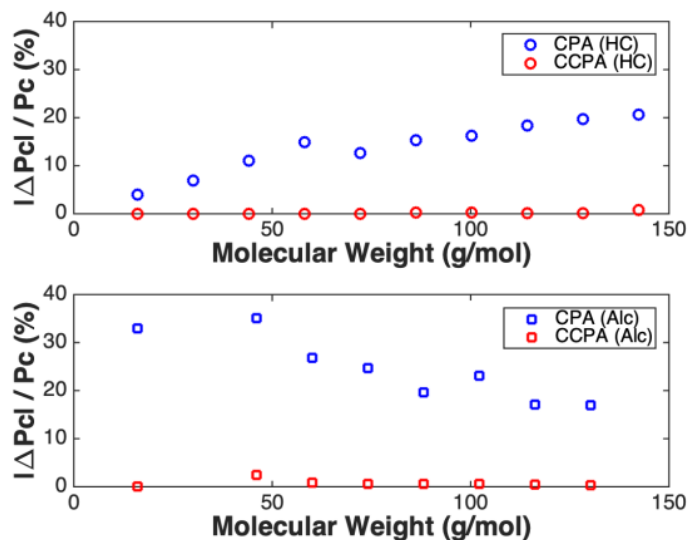

(b)
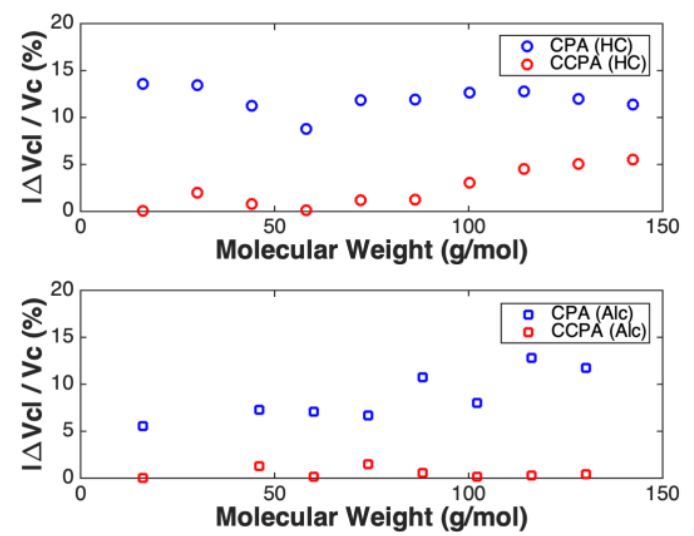

(c)

Figure 4: Deviations of the critical temperature (a), pressure (b) and density (c) as a function of the molecular weight of the alkanes (open circles) and alcohols (open squares). The deviations between the calculated and experimental critical properties were obtained with the CPA (blue circles and squares) and the CCPA (red circles and squares) EoS. Experimental data used in the comparison was taken from the DIPPR database..$^{55}$ 


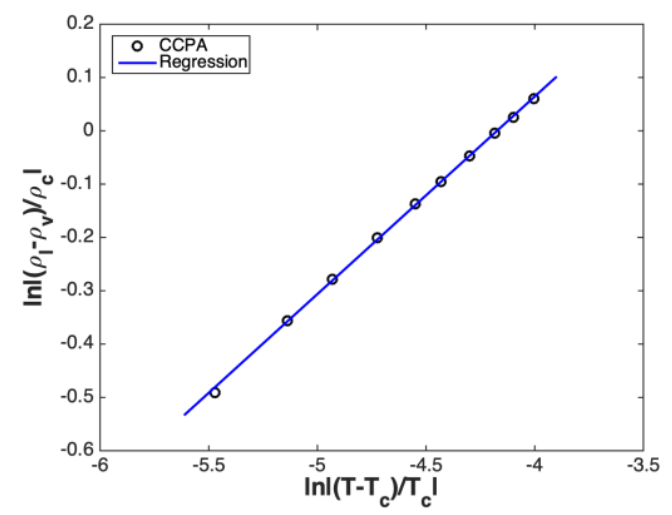

(a)

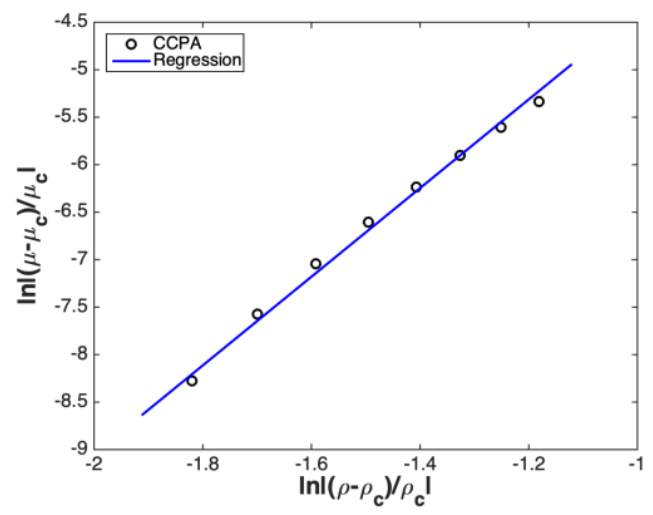

(b)

Figure 5: Critical exponents $\beta$ (a) and $\delta$ (b) calculated for 1-propanol using the $C C P A$ EoS.

\section{Application of the CCPA EoS to binary mixtures}

The estimation of the pure component parameters, along with the application of the combining and mixing rules described in the second section, allows the representation of the thermodynamic properties of mixtures. In this work, we have applied the classical $S R K$ and $C P A E O S$, as well as the and crossover version of the association model, to describe the phase equilibrium and critical properties of binary mixtures comprised of normal alkanes and 1-alkanols, specifically primary alcohols with straight alkane chains. In addition, we studied the phase behavior of some cross-associating systems, containing 1-alkanols, and solvating systems, containing carbon dioxide and a hydrogen bonding component. In both cases, only the classical and crossover models with the association term were used in the simulations.

\subsection{Binary mixtures of normal alkanes and 1-alkanols}

The first binary mixtures studied in this work were the methanol/n-hexane to methanol/n-decane systems. We have evaluated both phase equilibrium calculations in the vapor-liquid (VLE) and liquid-liquid (LLE) region, as well as the critical lines of these systems and compared the results with experimental data.

Figure 6 shows the experimental VLE (Figure 6a) and LLE (Figure 6b) data of methanol/n-hexane for conditions far away from the critical points and the calculated curves with SRK, $C P A$ and $C C P A$ EoS. In the case of the VLE calculations, the results were obtained for a temperature of $318.15 \mathrm{~K}$, while for the LLE diagram was correlated at 1 bar. In both cases, it is seen the crossover and classical CPA are superior to the cubic one and behave similarly, indicating that the fluctuations are negligible and that $C C P A$ reduces to $C P A$.

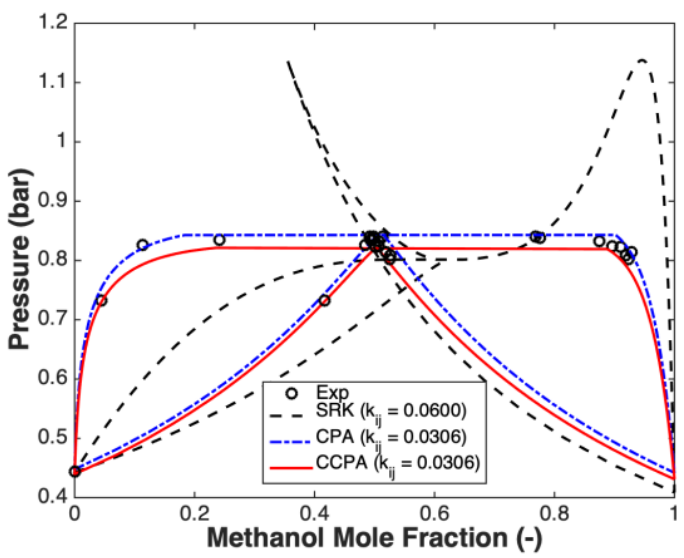

(a)

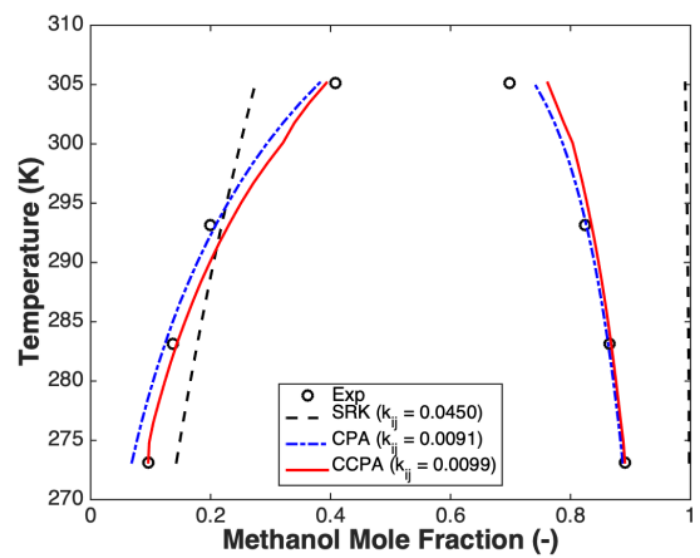

(b)

Figure 6: Experimental VLE at $318.15 \mathrm{~K}^{45}$ (a) and LLE at $1 \operatorname{bar}^{46}$ (b) for the methanol/n-hexane mixture. The simulated dashed black lines, dash-dotted blue lines and solid red lines correspond to SRK, CPA and CCPA, respectively. 


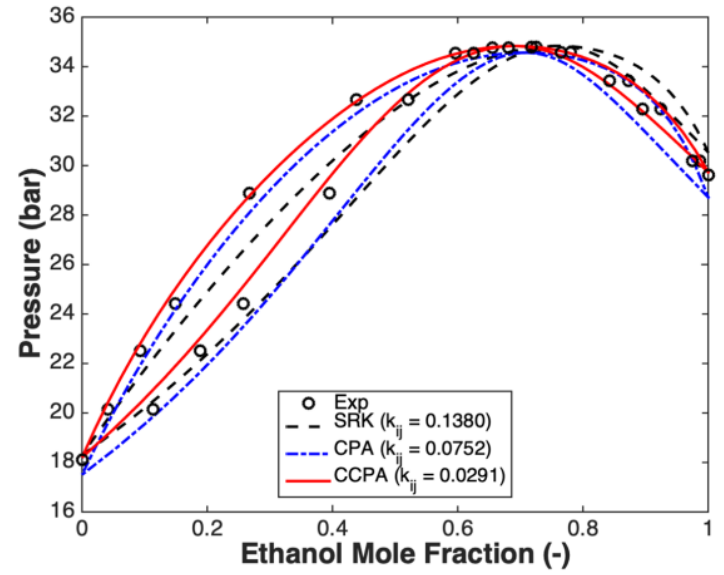

(a)

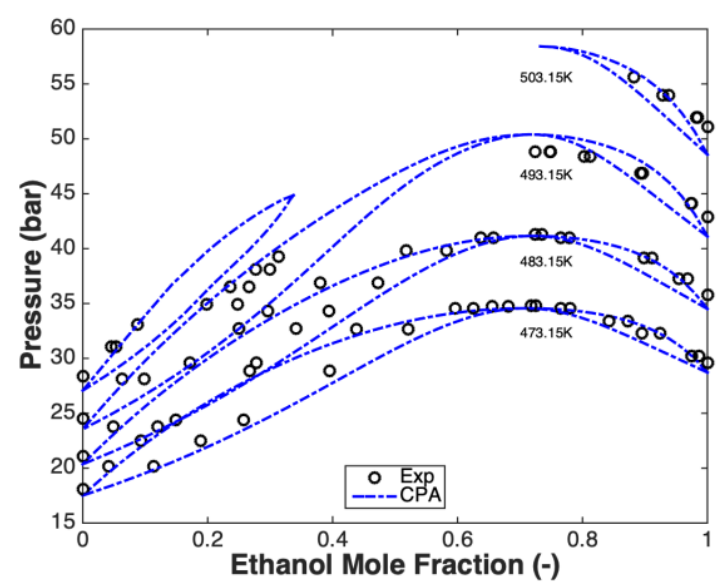

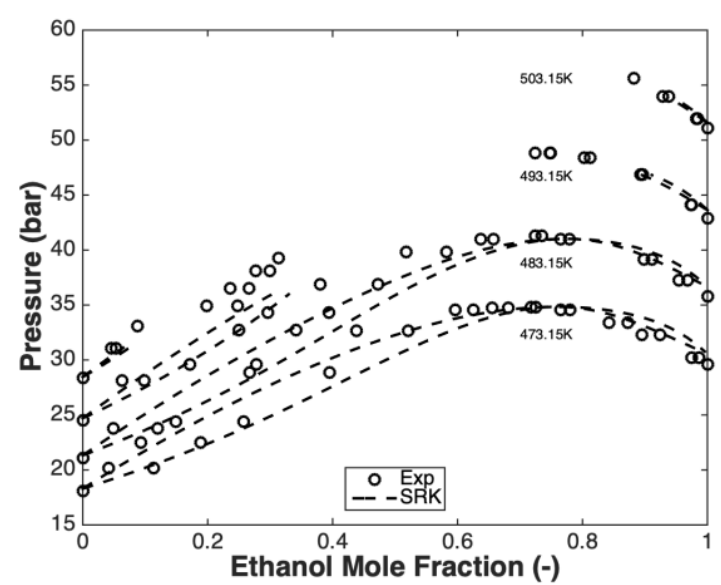

(b)

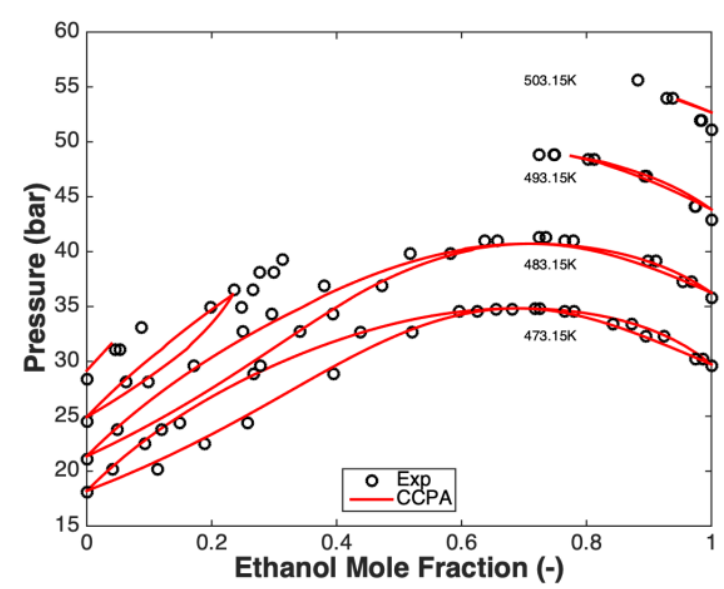

(c) (d)

Figure 7: Experimental near-critical VLE data for the ethanol/n-hexane ${ }^{47}$ system. The calculated dashed black lines, dashdotted blue lines and solid red lines were obtained with $S R K, C P A$ and $C C P A$, respectively. Figure (a) shows the comparison of the three models for a temperature of $473.15 \mathrm{~K}$, while figures (b) to (d) show the performance of each model for temperatures ranging from 473.15 to $500.15 \mathrm{~K}$. The binary interaction parameters from (a) were used in all figures.

The association term is crucial for the correct description of the systems at temperatures far away from the critical one. Figure 6 indicates that the attempt to correlate the data with the cubic model yields incorrect phase splits in the VLE diagram (Figure 6a) and much higher compositions of the associating component in the alcohol rich phase Figure (Figure 6b), in the case of LLE. Both behaviors are well known and widely discussed in the literature. ${ }^{3}$

On the other hand, as the critical point is approached, the introduction of the density fluctuations becomes important for the correct representation of the phase equilibrium properties of mixtures, while the association term becomes less significant. Figure $7 \mathrm{a}$ shows the calculated and experimental data of the VLE diagram of ethanol/n-hexane at $473.13 \mathrm{~K}$, while Figures $7 \mathrm{~b}$ to $7 \mathrm{~d}$ presents the phase diagram for several temperatures up to $500.15 \mathrm{~K}$ calculated with $S R K, C P A$ and CCPA, respectively. It is possible to conclude from the figure that the classical models require higher binary interaction parameters to correlate the experimental the vapor compositions and bubble point pressures. Yet, the phase envelopes simulated with $S R K$ are smaller than the experimental ones, while $C P A$ results in larger two-phase regions. The comparison of the results with experimental data shows that CCPA is superior to both models in the correlation of the VLE data as temperature increases. 
At the critical point, where the correlation length goes to infinity, the effect of the density inhomogeneities is substantial. Figures 8 and 9 show the critical curves of methanol/n-alkane and ethanol/n-hexane systems. The calculations were pure predictions, as no binary interaction parameters were used. This was done in order to appropriately compare the three models, since the $k_{i j}$ does not correct the models close to pure component conditions.

The representation of the methanol/n-alkane system with the $S R K$ and $C P A E O S$ was thoroughly evaluated in a previous work. ${ }^{11}$ It was demonstrated that the traditional parameterization procedure of CPA causes an overprediction of the critical point, resulting in larger deviations, as seen in figures $8 \mathrm{~b}$ and 9 . Rescaling the parameters of the model improves the description of the critical data, but deteriorates the representations far away from the critical point, in special the LLE diagrams. On the other hand, the cubic model is unable to capture the trend in the experimental data, which seems to indicate that the hydrogen bonding term is still important even at critical conditions. This behavior is also corroborated by the results from the CCPA EoS, which corrects the overprediction of the classical model and is superior to both the classical cubic and association EoS. In fact, it is the only model capable of representing the phase behavior of the methanol/n-hexane both the far away from and close to the critical point. It is important to note, that similar results were obtained by $\mathrm{Xu}$ et al., ${ }^{36}$ while using a slightly different version of the crossover CPA EoS.

The comparison of the three models for methanol/nalkane mixtures indicates better representation of the experimental data is obtained for the lighter n-alkanes. On the other hand, the deviations increase for heavier molecules, which is related to the increase in the asymmetry of the weight of the components in the mixture.

Finally, the classical and crossover EoS were applied in the description of the critical locus of the ethanol/nhexane system (Figure 9). In this case, the pressurecomposition (Figure 9a) and temperature-composition (Figure 9b) diagrams show that the classical models predict higher critical properties for the mixture or are unable to get a good description of the minimum critical temperature. Nonetheless, the use of the association term and recursive procedure to account for density fluctuations results in a precise prediction of the critical behavior of the system.

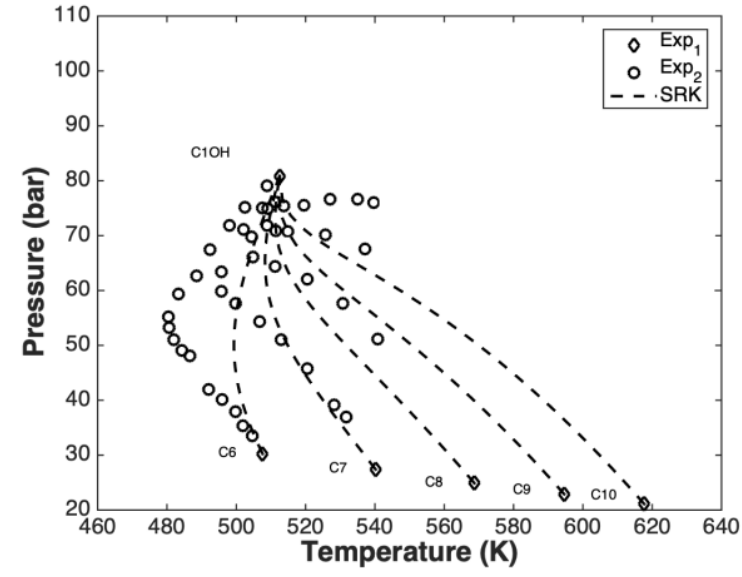

(a)

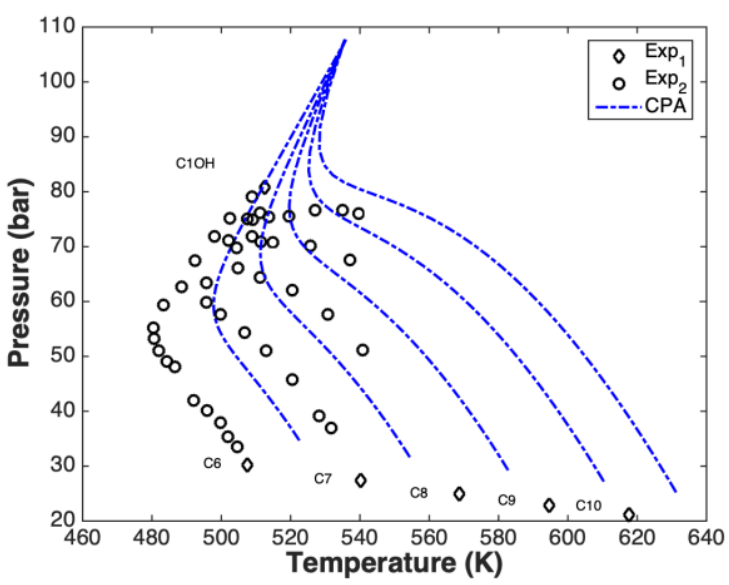

(b)

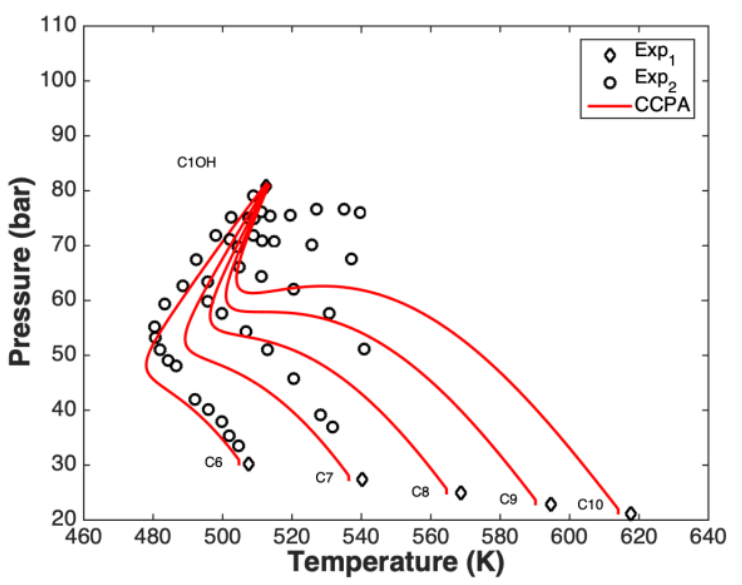

(c)

Figure 8: Experimental critical loci of methanol/n-hexane to methanol/n-decane mixtures. ${ }^{48}$ The predicted curves $(\mathrm{kij}=0)$ were obtained with $S R K$ (a), CPA (b) and CCPA (c). 


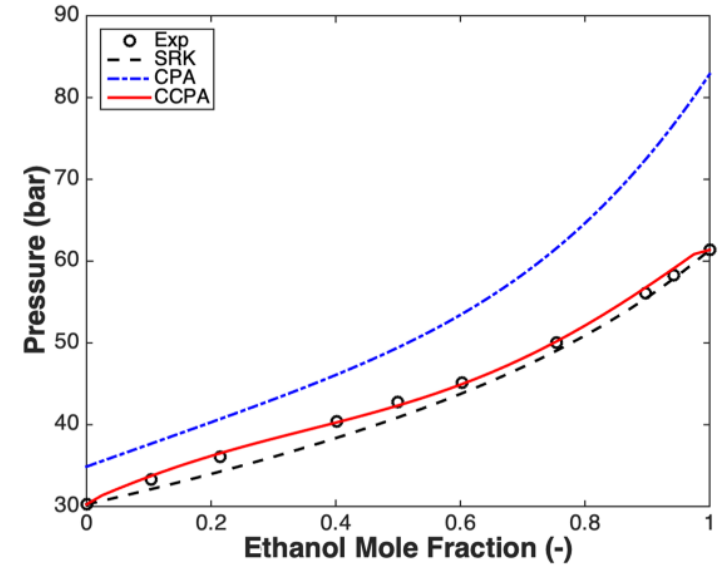

(a)

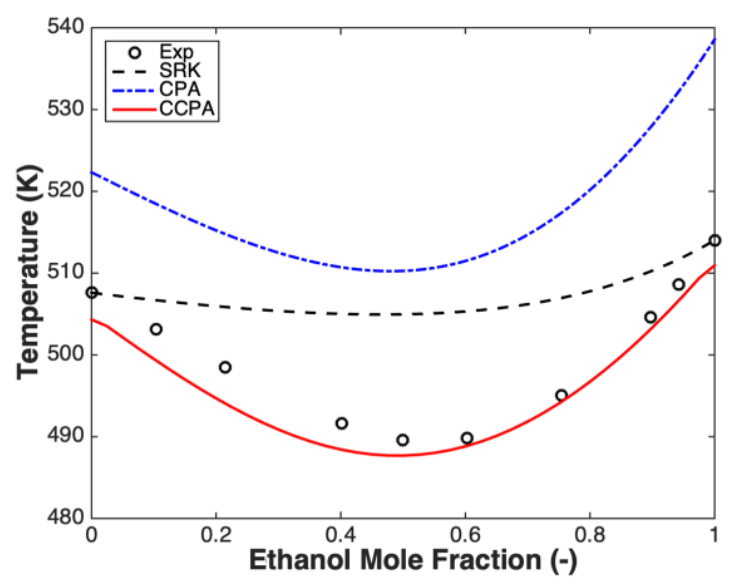

(b)

Figure 9: Experimental critical temperature (a) and pressure (b) as a function of ethanol mole fraction for the ethanol/n-hexane mixture..$^{48}$ The predicted $(\mathrm{kij}=0)$ dashed black lines, dash-dotted blue lines and solid red lines correspond to $S R K, C P A$ and $C C P A$, respectively.

\subsection{Binary mixtures of cross-associating 1-alkanols}

Calculations for cross-associating systems composed of two alcohols, i.e. methanol/1-propanol and methanol/1-butanol, were also performed in this work, using only the models with an association term. This is due to the fact that poor behavior has been observed when cubic models with traditional mixing rules are applied to describe cross-associating systems. ${ }^{3}$ The same is valid for solvating mixture, therefore it is not used in the next section as well.

The importance of these calculations is to test the models for cross-associating systems. As mentioned previously, we have used the CRI combining rule to calculate the interaction between the different associating energies and volumes. As seen in Figure 10, the trends of the critical pressures and temperatures are similar, although $C P A$ yields larger deviations than the crossover model. These results show that the recursive procedure yields a quantitative prediction of the experimental data by reducing the critical temperatures and pressures of the simulated values. However, the similarity in the trends indicates that the recursive procedure does not change the features of the classical $E o S$, implying that, in addition to the renormalization group correction, the association term and the combining rule are fundamental for the correct representation of the experimental data.

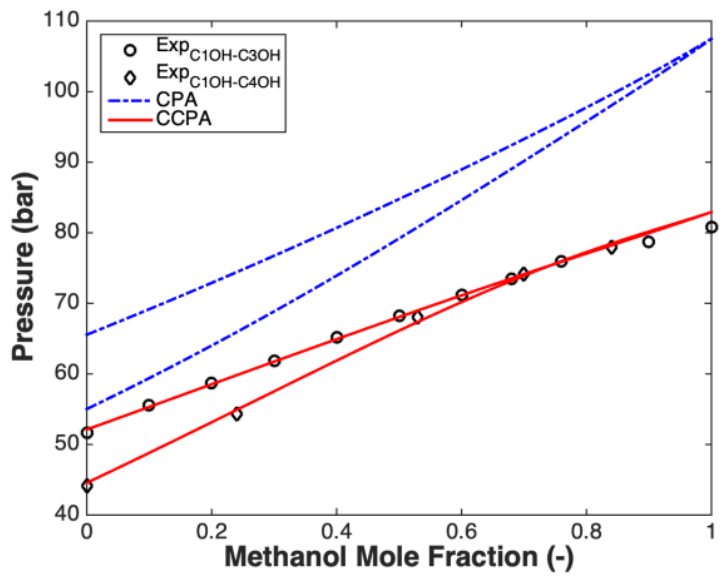

(a)

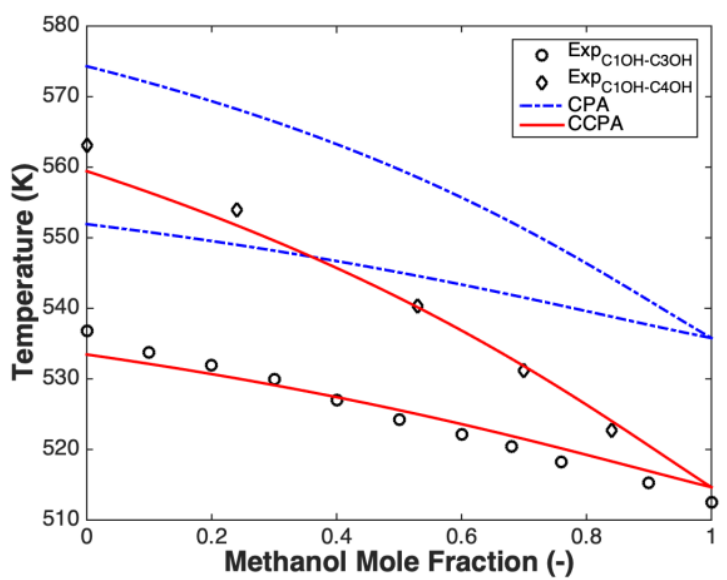

(b)

Figure 10: Experimental critical temperature (a) and pressure (b) as a function of methanol mole fraction for the methanol/1-propanol ${ }^{49}$ and methanol/1-butanol ${ }^{50}$ mixtures. The simulated dash-dotted blue lines and solid red lines were obtained using the CPA and CCPA EoS, respectively. 


\subsection{Binary mixture of carbon dioxide and water}

Finally, we investigated systems containing carbon dioxide and an associating component, specifically the $\mathrm{CO}_{2} /$ methanol and $\mathrm{CO}_{2} /$ water systems. Different from the previous subsection, the solvation rule was applied to model the interaction between the non-associating component $\left(\mathrm{CO}_{2}\right)$ and the hydrogen-bonding one (water or methanol).

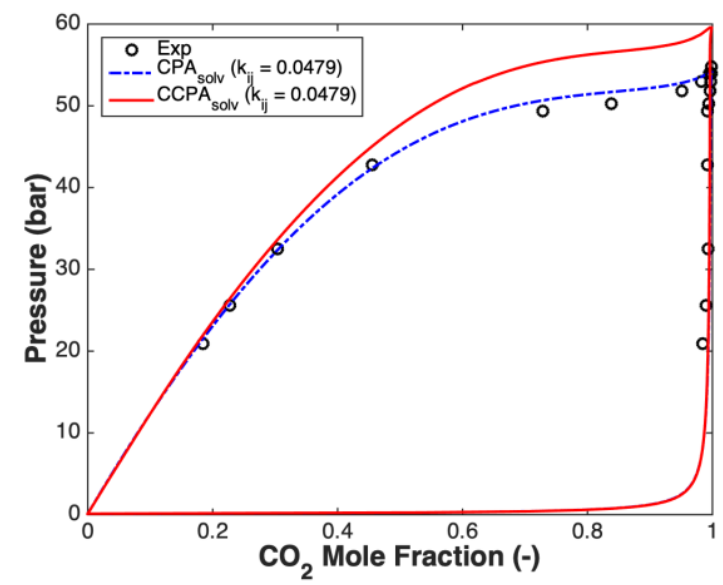

(a)

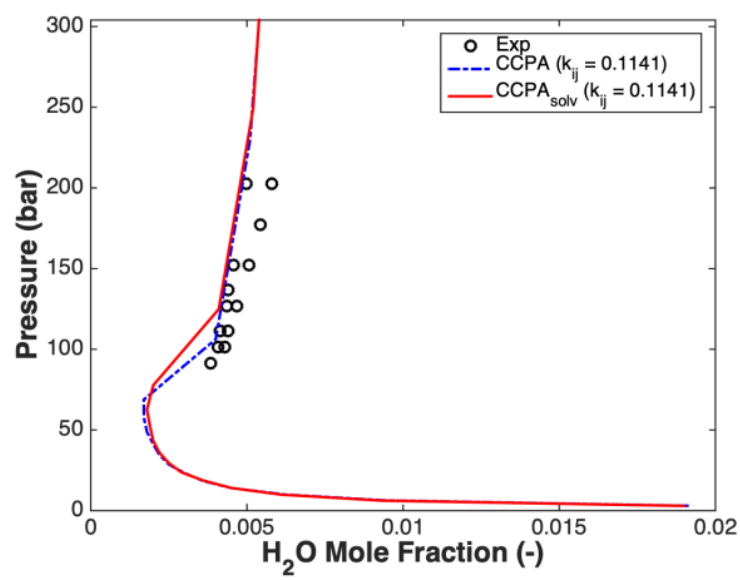

(b)

Figure 11: Experimental VLE data of carbon dioxide and methanol at $290 \mathrm{~K}^{51}$ (a) and carbon dioxide and water at $308 \mathrm{~K}^{52}$ (b). The calculated dash-dotted blue lines and solid red lines were obtained using the $C P A$ and $C C P A$ $E o S$, respectively.

The results for the calculations of the VLE region of the mixtures containing carbon dioxide and an associating component indicate that the models behave similarly. However, CCPA performs better for the mixture with water in comparison to the mixture with methanol. This is an indication that the solvation approach is important for yielding the correct behavior of the fluids; nonetheless, the pure component properties for methanol should be reevaluated in order to yield a better representation of the experimental data at higher pressures.

Although accurate representation of the fluids in conditions far from critical was attained with the two models, large deviations for the critical lines are observed (Figure 12). The incorporation of the density fluctuation into the classical model via the recursive procedure proposed by White corrected the CPA EoS close to the critical point of the pure hydrogen-bonding component; nevertheless, as the composition of carbon dioxide increases, the crossover model behaves more similarly to the classical one. The large deviations observed in the critical diagrams might be related to the utilization of $k_{i j}$ regressed for the classical model in sub-critical conditions, or a temperature-independent $\beta$ parameter in the solvation model, fitted to experimental VLE data at temperatures far away from the critical point. In fact, the precise thermodynamic modeling of $\mathrm{CO}_{2}$ and hydrogen bonding systems over a wide range of conditions is a challenging task, and different methods are suggested in the literature.

In the case of classical models, Polishuk et al. ${ }^{56}$ introduced interaction parameters for the repulsive term $\left(l_{i j}\right)$ in different $E O S$ for describing sub-critical and critical behavior of mixtures containing $\mathrm{CO}_{2}$ and alkanols. A different approach was applied by Koláŕ and Kojima, ${ }^{57}$ using the Predictive SRK EoS (PSRK). PSRK is a combination of local mixing rules, the UNIFAC group contribution method and the $S R K$ with Mathias-Copeman alpha-function, which considerably increases the versatility of the cubic model and allows the representation of complex systems over a wide range of conditions. Nonetheless, for the case of methanol and $\mathrm{CO}_{2}$, the authors modified the original parameters of the model, correlating it to the experimental critical data. The original model overpredicted the measured points and predicted a Type $\mathrm{V}$ phase behavior, similar to the one given by $C P A$ in Figure 12a. The correlated model was able to describe the correct phase behavior of the mixture, i.e. Type I, and to reduce significantly the deviations with respect to the experimental data. In this work, the CCPA EoS is not capable of correctly describing the experimental critical points; nonetheless, it yields the correct phase behavior (Type I), since the critical points of the two pure components are connected by a single continuous critical line.

One attempt to represent the critical behavior of solvating systems with crossover models is found in the literature. Llovell et al. ${ }^{15}$ obtained excellent results with the crossover Soft-SAFT EOS using a model that takes 
into account the quadrupolar moment of the nonassociating molecule. In this case, the solvation method is replaced by a quadrupolar model, in order to correctly describe the behavior of $\mathrm{CO}_{2}$ in mixtures with alkanols. Apart from the introduction of a quadrupolar term in the crossover EoS, better results may be achieved with a different implementation of the renormalization procedure for mixtures.

In this work, it is assumed that a mixture's thermodynamic potential has the same universal form as the one-component fluid. This implementation is referred to as the isomorphism assumption, and the global density of the system is used as the isomorphic variable. A different approach is called the "phase-space cell approximation", originally suggested by Wilson. ${ }^{16,17}$ The properties of the mixture are determined by calculating the density fluctuations of each component independently of the other components density fluctuations. Tang and Gross $^{37}$ compared both methods using a crossover $P C$ SAFT EOS for describing binary alkane-alkane systems and mixtures with $\mathrm{CO}_{2}$ and $\mathrm{H}_{2} \mathrm{~S}$. The authors concluded that the phase-space cell approximation assumption yields slightly superior results in comparison to the isomorphic approximation. Nonetheless, the computation time required for the calculations rises dramatically with the number of components, ${ }^{38}$ making it less suitable for practical applications.

\section{Conclusions}

In this work, we have applied the CCPA EoS to represent the phase equilibrium and critical properties of n-alkanes and alcohols, and compared the results with the classical EoS, namely $S R K$ and $C P A$.

We have obtained new pure component parameters for the substances studied, and with these parameters, the model is able to precisely predict the saturated properties of the species studied, as well as the critical point, although an increase in the deviations of the vapor pressure is observed in comparison with the CPA EoS. The crossover equation was also applied in the calculation of two critical exponents and values close to the universal ones were found, indicating the nonanalytical nature of the thermodynamic model.

The application of the CCPA $E O S$ to describe the VLE and $L L E$ diagrams of the binary mixtures far away from the critical point showed that the crossover model behaves similarly to $C P A$, and it is superior to the cubic model. Additionally, as the critical point is approached, CCPA also yields better results than both classical formulations, except for systems containing carbon dioxide and a hydrogen-bonding molecule. Besides, large deviations were observed in the description of the critical lines of solvating mixtures. The reason for this behavior might be related to the large quadrupolar moment of $\mathrm{CO}_{2}$, which is not taken into account by the model developed in this work.

Finally, the models were also used in the calculation of the critical lines of the alcohol/n-alkane, alcohol/alcohol binary mixtures. It was seen that, apart from the solvating mixtures, the use of the association term and the recursive procedure for introducing density fluctuations into the classical model results in a precise prediction of the experimental critical pressures and temperatures.

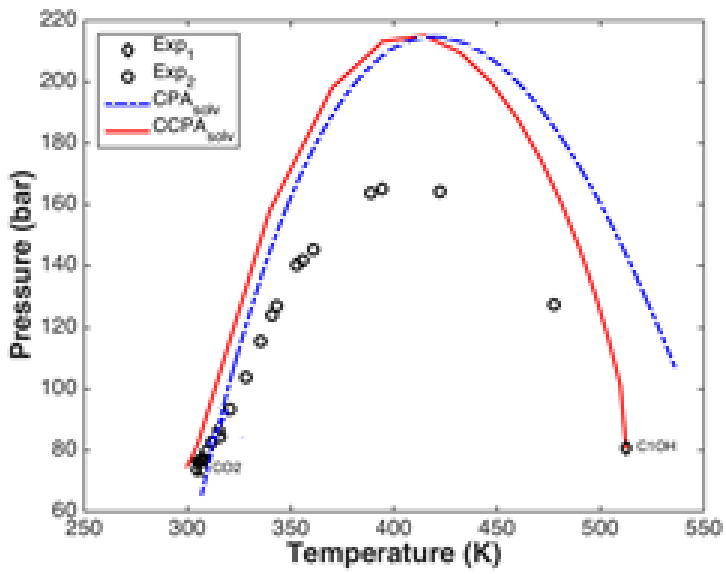

(a)

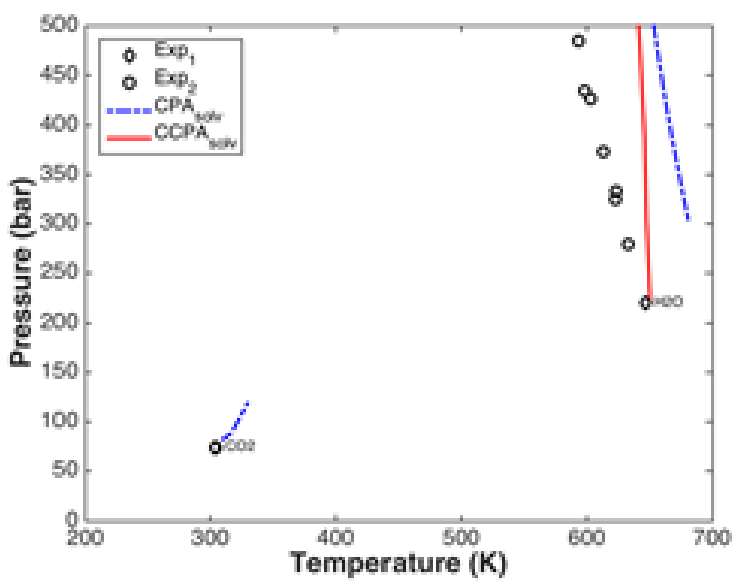

(b)

Figure 12: Experimental critical loci of the carbon dioxide/methanol ${ }^{53}$ (a) and carbon dioxide/water ${ }^{54}$ (b) systems. The calculated dash-dotted blue lines and solid red lines were obtained using the $C P A$ and $C C P A E O S$, respectively. 


\section{Acknowledgment}

The authors would like to express their gratitude to the program "Science without Borders" for the $\mathrm{PhD}$ scholarship of Andre Pinto Coelho Muniz Vinhal.

\section{List of symbols}

\section{Abbreviations}

AAD: average absolute deviation $\left(\frac{100}{N_{\text {exp }}} \sum\left|\frac{x_{c a l c}-x_{\text {exp }}}{x_{\text {exp }}}\right|\right)$

Alc: Alcohol(s)

$C P A$ : Cubic-Plus-Association equation of state

CCPA: Crossover Cubic-Plus-Association equation of state

DIPPR: The Design Institute for Physical Properties

$E o S$ : equation of state

$\mathrm{HC}$ : Hydrocarbon(s)

$L L E$ : Liquid-liquid equilibrium

NIST: National Institute of Standards and Technology

$S R K$ : Soave-Redlich-Kwong equation of state

$V L E$ : Vapor-liquid equilibrium

\section{Roman letters}

$a$ : Energy parameter of $C P A$

$b$ : Size-related parameter of $C P A$

$c_{1}$ : Parameter of $C P A$

$f$ : Free energy density

$g$ : Simplified radial distribution function

$G$ : Functions of the modified free energy density

$k_{b}$ : Boltzmann constant

$k_{i j}$ : Binary interaction coefficient

$K_{n}$ : Recursive procedure coefficient

$L$ : Cutoff length

$N$ : Number of experimental points

$P:$ Pressure

$R$ : Ideal gas constant

$T$ : Temperature

$v$ : Molar volume

$x$ : Mole fraction or liquid phase mole fraction

$X_{A_{i}}:$ Monomer fraction

$y$ : Vapor phase mole fraction

\section{Greek letters}

$\alpha$ : Attractive term correction in the recursive procedure

$\beta$ : Critical exponent

$\beta^{A_{i} B_{j}}$ : Association volume parameter

$\delta$ : Critical exponent

$\Delta^{A_{i} B_{j}}$ : Association strength

$\varepsilon^{A_{i} B_{j}}$ : Association energy parameter

$\rho$ : Molar density

$\phi$ : Initial shortest wavelength parameter

$\Omega$ : Contributions of the density fluctuations

\section{Super/subscripts}

alc: Alcohol rich phase

$c$ : Critical property

$\mathrm{C} 1 \mathrm{OH}$ : Composition of methanol

calc: Calculated property

exp: Experimental property

$h c$ : Hydrocarbon rich phase

$i, j$ : Component index

liq: Liquid phase property

$n$ : Iteration index

sat: Saturated property

vap: Vapor phase property

\section{Supporting information available:}

Description of the algorithm used in the critical point calculations with the crossover EoS.

\section{References}

(1) Hendriks, E.; Kontogeorgis, G. M.; Dohrn, R.; de Hemptinne, J.-C.; Economou, I. G.; Žilnik, L. F.; Vesovic, V. Industrial Requirements for Thermodynamics and Transport Properties. Ind. Eng. Chem. Res. 2010, 49, 22, 11131-11141.

(2) Wei, Y.; Chen, Z. J.; Satyro, M.; Dong, C.; Deng, H. Compositional Simulation Using the Advanced PengRobinson Equation of State, SPE Reservoir Simulation Symposium, Woodlands, Texas, USA, 2011.

(3) Kontogeorgis; G. M., Folas, G. K. Thermodynamic Models for Industrial Applications. From Classical and Advanced Mixing Rules to Association Theories. New York: Wiley \& Sons, 2010.

(4) Anisimov, M. A.; Sengers, J. V. Critical and Crossover Phenomena in Fluids and Fluid Mixtures. Supercritical Fluids, Netherlands, Springer, 2000, 89-121.

(5) Anisimov, M. A.; Sengers, J. V. Critical Region. Equations of State for Fluids and Fluid Mixtures. Elsevier, Amsterdam, 2000.

(6) Kontogeorgis, G. M.; Michelsen, M. L.; Folas, G. K.; Derawi, S.; von Solms, N.; Stenby, E. H. Ten Years with the CPA (Cubic-Plus-Association) Equation of State. Part 1. Pure Compounds and Self-Associating Systems. Ind. Eng. Chem. Res., 2006, 45, 14, 48554868.

(7) Kontogeorgis, G. M.; Michelsen, M. L.; Folas, G. K.; Derawi, S.; von Solms, N.; Stenby, E. H. Ten Years with the CPA (Cubic-Plus-Association) Equation of State. Part 2. Cross-Associating and Multicomponent Systems. Ind. Eng. Chem. Res., 2006, 45, 14, 4869- 
4878.

(8) Kontogeorgis, G. M.; Voutsas, E. C.; Yakoumis, I. V.; Tassios, D. P. An Equation of State for Associating Fluids. Ind. Eng. Chem. Res., 1996, 35, 11, 4310-4318.

(9) Soave, G. Equilibrium Constants from A Modified Redlich-Kwong Equation Of State. Chem. Eng. Sci., 1972, 27, 6, 1197-1203.

(10) Wertheim, M. S. Fluids with Highly Directional Attractive Forces. I. Statistical Thermodynamics. $J$. Stat. Phys., 1984, 35, 1-2, 19-34.

(11) Vinhal, A. P. C. M.; Yan, W.; Kontogeorgios, G. M. Evaluation of Equations of State for Simultaneous Representation of Phase Equilibrium and Critical Phenomena. Fluid Phase Equilib., 2007, 437, 140-154.

(12) Wyczalkowska, A. K.; Sengers, J. V.; Anisimov, M. A. Critical fluctuations and the equation of state of van der Waals. Physica A, 2004, 334, 3-4, 482-512.

(13) Kiselev, S. B.; Cubic crossover equation of state. Fluid Phase Equilib., 1998, 147, 1-2, 7-23.

(14) Sengers, J. V.; Sengers, J. M. H. L. Thermodynamic behavior of fluids near the critical point. Annu. Rev. Phys. Chem., 1986, 37, 189-222.

(15) Llovell, F.; Vega, L. F. Global Fluid Phase Equilibria and Critical Phenomena of Selected Mixtures Using the Crossover Soft-SAFT Equation. J. Phys. Chem. B, 2006, 110, 3, 1350-1362.

(16) Wilson, K. G. Renormalization Group and Critical Phenomena. I. Renormalization Group and the Kadanoff Scaling Picture. Phys. Rev. B, 1971, 4, 9, 3174-3183.

(17) Wilson, K. G.; Fisher, M. E. Critical Exponents in 3.99 Dimensions. Phys. Rev. Lett., 1972, 28, 240-243.

(18) Binney, J. J.; Dowrick, N. J.; Fisher, A. J.; Newman, M. E. J. The Theory of Critical Phenomena: An Introduction to the Renormalization Group. Clarendon, Oxford,1992.

(19) Ma, S.-K.; Modern Theory of Critical Phenomena. New York, Westview Press, 2000

(20) Forte, E.; Llovell, F.; Vega, L. F.; Trusler, J. P. M.; Galindo, A. Application of a Renormalization-Group Treatment to the Statistical Associating Fluid Theory for Potentials of Variable Range (SAFT-VR). J. Chem. Phys., 2011, 134, 154102, 1-15

(21) Chen, Z. Y.; Albright, P. C.; Sengers, J. V. Crossover from Singular Critical to Regular Classical Thermodynamic Behavior of Fluids. Phys. Rev. A, 1990, 41, 6, 3161-3177.

(22) Chen, Z. Y.; Abbaci, A.; Tang, S.; Sengers, J. V. Global thermodynamic behavior of fluids in the critical region.
Phys. Rev. A, 1990, 42, 4470-4484.

(23) Kiselev, S. B.; Friend, D. G. Cubic Crossover Equation of State for Mixtures. Fluid Phase Equilib., 1999, 162, 1-2, 51-82.

(24) Kiselev, S. B.; Ely, J. F. Simplified crossover SAFT equation of state for pure fluids and fluid mixtures. Fluid Phase Equilib., 2000, 174, 1-2, 93-113.

(25) McCabe, C.; Kiselev, S. B. Application of Crossover Theory to the SAFT-VR Equation of State: SAFT-VRX for Pure Fluids. Ind. Eng. Chem. Res., 2004, 43, 28392851

(26) Sun, L.; Zhao, H.; Kiselev, S. B.; McCabe, C. Predicting Mixture Phase Equilibria and Critical Behavior Using the SAFT-VRX Approach. J. Phys. Chem. B, 2005, 109, 9047-9058

(27) Salvino, L. W.; White, J. A. Calculation of density fluctuation contributions to thermodynamic properties of simple fluids. J. Chem. Phys., 1992, 96, 4559-4568.

(28) White, J. A.; Zhang, S. Renormalization group theory for fluids. J. Chem. Phys., 1993, 99, 2012-2019.

(29) White, J. A.; Zhang, S. Renormalization theory of nonuniversal thermal properties of fluids. J. Chem. Phys., 1995, 103, 1922-1928.

(30) White, J. A.; Zhang, S. Renormalization Group Theory for Fluids to Greater Density Distances from the Critical Point. Int. J. of Thermophys., 1998, 19, 4, 10191027.

(31) Vinhal, A. P. C. M.; Kontogeorgis, G. M.; Wei, Y. Application of a Crossover Equation of State to Describe Phase Equilibrium and Critical Properties of n-Alkanes and Methane/n-Alkane Mixtures. J. Chem. Eng. Data, 2018, 63, 4, 981-993.

(32) Llovell, F. Accurate Description of the Critical Region by a Molecular-Based Equation of State with a Crossover Treatment. Doctoral Dissertation, Universitat Autonoma de Barcelona, 2006.

(33) Llovell, F.; Vega, L. F. Phase Equilibria, Critical Behavior and Derivative Properties of Selected nAlkane/n-Alkane and n-Alkane/1-Alkanol Mixtures by the Crossover Soft-SAFT Equation of State. $J$. Supercrit. Fluids, 2007, 41, 2, 204-216.

(34) Llovell, F.; Pamies, J. C.; Vega, L. F. Thermodynamic properties of Lennard-Jones chain molecules: Renormalization-group corrections to a modified statistical associating fluid theory. J. Chem. Phys., 2004, 121, 10715-10724.

(35) Xu, X.-H.; Duan, Y.-Y. Crossover CPA Equation of State for Associating Fluids. Fluid Phase Equilib., 2010, 290, 1-2, 148-152.

(36) Xu, X.-H.; Duan, Y.-Y.; Yang, Z. Prediction of the 
CriticaI Properties of Binary Alkanol + Alkane Mixtures Using a Crossover CPA Equation of State. Fluid Phase Equilib., 2011, 309, 2, 168-173.

(37) Tang, X.; Gross, J. Renormalization-Group Corrections to the Perturbed-Chain Statistical Associating Fluid Theory for Binary Mixtures. Ind. Eng. Chem. Res., 2010, 49, 19, 9436-9444.

(38) Lue, L.; Prausnitz, J. M. Thermodynamics of Fluid Mixtures Near to and Far from the Critical Region. AIChE Journal, 1998, 44, 6, 1455-1466

(39) Wilson, K. G. Renormalization Group and Critical Phenomena. II. Phase-Space Cell Analysis of Critical Behavior. Phys. Rev. B, 1971, 4, 9, 3184-3205

(40) Huang, S. H.; Radosz, M. Equation of State for Small, Large, Polydisperse, and Associating Molecules. Ind. Eng. Chem. Res., 1990, 29, 11, 2284-2294.

(41) Kontogeorgis, G. M.; Yakoumis, I. V.; Meijer, H.; Hendriks, E.; Moorwood, T. Multicomponent Phase Equilibrium Calculations for Water-Methanol-Alkane Mixtures. Fluid Phase Equilib., 1999, 158-160, 201209.

(42) Folas, G. K. Modeling of Complex Mixtures Containing Hydrogen Bonding Molecules. Doctoral Dissertation. Technical University of Denmark, Copenhagen, 2007.

(43) Yakoumis, I. V.; Kontogeorgis, G. M.; Voutsas, E. C.; Tassios, D. P. Vapor-Liquid Equilibria for Alcohol/Hydrocarbon Systems Using the CPA Equation of State. Fluid Phase Equilib., 1997, 130, 31-47.

(44) Janecek, J.; Paricaud, P.; Dicko, M.; Coquelet, C. A Generalized Kiselev Crossover Approach Applied to Soave-Redlich-Kwong Equation of State. Fluid Phase Equilib., 2015, 401, 16-26.

(45) Hongo, M.; Tsuji, T.; Fukuchi, K.; Arai, Y. VaporLiquid Equilibria of Methanol + Hexane, Methanol + Heptane, Ethanol + Hexane, Ethanol + Heptane, and Ethanol + Octane at 298.15K. J. Chem. Eng. Data, 1994, 39, 4, 668-691.

(46) Skrzecz, A. Critical Evaluation of Solubility Data in Binary Systems Formed by Methanol with nHydrocarbons. Thermochim. Acta, 1991, 182, 1, 123131.

(47) Seo, J.; Lee J.; Kim, H. Isothermal vapor-liquid equilibria for the system ethanol and n-hexane in the near critical region. Fluid Phase Equilib., 2001, 182, 12, 199-207.

(48) Liu, J.; Qin, Z.; Wang, G. Hou, X.; Wang, J. Critical Properties of Binary and Ternary Mixtures of Hexane + Methanol, Hexane + Carbon Dioxide, Methanol + Carbon Dioxide, and Hexane + Carbon Dioxide +
Methanol. J. Chem. Eng. Data, 2003, 48, 6, 1610-1613.

(49) Nazmutdinov, A. G.; Alekina, E. V.; Nesterova, T. N. Concentration dependences of the critical temperatures of binary mixtures of nonaqueous components. Russ. $J$. Phys. Chem. A., 2008, 82, 11, 1857-1862.

(50) Wang, L.; Han, K.; Xia, S.; Ma, P.; Yan, F. Measurement and correlation of critical properties for binary mixtures and ternary mixtures containing gasoline additives. J. Chem. Thermodyn., 2014, 74, 161-168.

(51) Hong, J. H.; Kobayashi, R. Vapor-liquid equilibrium studies for the carbon dioxide-methanol system. Fluid Phase Equilib., 1988, 41, 3, 269-276.

(52) King, M. B.; Mubarak, A.; Kim, J. D.; Bott, T. R. The mutual solubilities of water with supercritical and liquid carbon dioxides. J. Supercrit. Fluids, 1992, 5, 4, 296302.

(53) Brunner, E.; Hultenschmidt, W.; Schlichtharle, G. Fluid mixtures at high pressures IV. Isothermal phase equilibria in binary mixtures consisting of (methanol + hydrogen or nitrogen or methane or carbon monoxide or carbon dioxide). J. Chem. Therm., 1987, 19, 3, 273-291.

(54) Toedheide, K.; Franck, E. U. The two-phase region and the critical curve in the carbon dioxide + water system at pressures up to 3500 bars. Z. Physik. Chem., 1963, 37, 387-401.

(55) Rowley, R. L.; Wilding, W. V.; Oscarson, J. L.; Zundel, N.A.; Marshall, T. L.; Daubert, T. E.; Danner, R. P. DIPPR Data Compilation of Pure Compound Properties. Design Institute for Physical Properties, AIChE, New York, 2002.

(56) Polishuk, I.; Wisniak, J.; Segura, H. Simultaneous prediction of the critical and sub-critical phase behavior in mixtures using equations of state II. Carbon dioxideheavy n-alkanes. Chem. Eng. Sci., 2003, 58, 12, 25292550.

(57) Kolár, P.; Kojima, K. Prediction of critical points in multicomponent systems using the PSRK group contribution equation of state. Fluid Phase Equilib. 1996, 118, 175-200. 


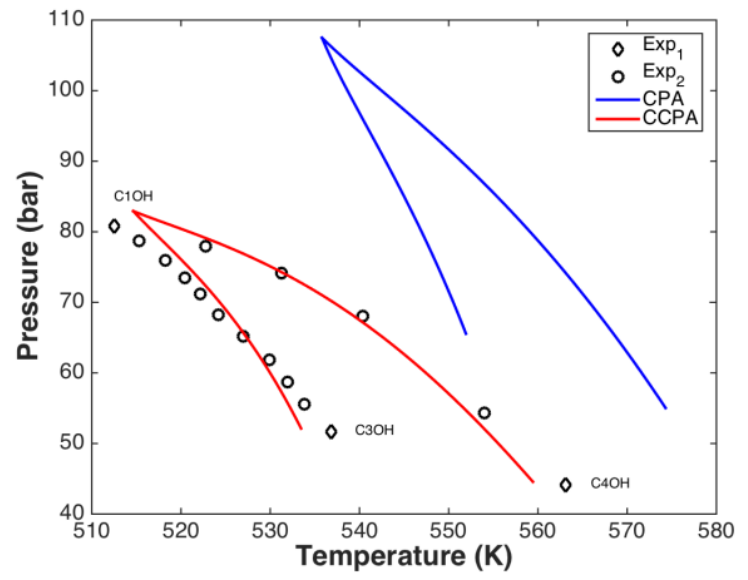

For table of contents only. 